\title{
Digit ratio (2D:4D) is associated with breast cancer
}

\section{Patrícia Helena Costa Mendes"1, Ana Carolina Campos Gomes ${ }^{2}$, Priscila Bernadina Soares ${ }^{3}$, Eduardo Gonçalves', Clayton Paraíso Macedo4, Marise Fagundes Silveira1, Daniella Reis Martelli', Lívia Máris Ribeiro Paranaíba'1, Hercílio Martelli-Júnior ${ }^{1}$}

\author{
${ }^{1}$ Department of Health Science Program, State University of Montes Claros, Minas Gerais State, Brazil \\ ${ }^{2}$ Dental School, State University of Montes Claros, Minas Gerais State, Brazil \\ ${ }^{3}$ Department of Medical Oncologist, Oncology Department of Santa Casa, Minas Gerais State, Brazil \\ ${ }^{4}$ Medical School, State University of Montes Claros, Minas Gerais State, Brazil
}

Received March 20, 2016; Revised June 16, 2016; Accepted June 20, 2016; Published Online July 16, 2016

\section{Original Article}

\begin{abstract}
Purpose: Digit ratio (2D:4D) has been considered as a proxy biomarker for prenatal hormonal exposure and may represent an individual's predisposition to breast cancer. The purpose of the present study is to investigate whether there is a link between digit ratio and breast cancer in a Brazilian population. Methods: Digital measurements of the lengths of the index and ring fingers of both hands were obtained from women with breast cancer $(\mathrm{n}=100)$ and age-matched controls $(\mathrm{n}=$ 100) using a digital Vernier calliper. Mean digit ratios of right hands, left hands, and right minus left hand 2D:4D (DR-L) were compared between both groups. Data were analysed by the Student's $t$-test for unpaired samples, Mann-Whitney test, and Spearman`s correlation with a significance level of 5\%. Results: The patients with breast cancer presented significantly higher right and left 2D:4D (both $p<0.001$ ) and higher DR-L ( $p=0.032)$ than controls. Among breast cancer cases, there was a significantly negative correlation between left 2D:4D and age diagnosed with breast cancer $(p=0.018)$. Conclusion: Digit ratio offers a valid retrospective biomarker of action of prenatal hormones and might be associated with breast cancer risk and age at onset of breast cancer. It suggests that higher exposure or sensitivity to prenatal oestrogen might be associated with a higher risk of breast cancer and with earlier onset of the disease.
\end{abstract}

Keywords: Digit Ratio, 2D:4D, Intrauterine Oestrogen, Breast Cancer

\section{Introduction}

A new focus of adult cancer research is on the in utero and early life periods. Breast cancer has concentrated the most researches about factors associated with intrauterine period and early life. ${ }^{1}$ Intrauterine environmental exposure to endogenous or exogenous hormones, notably oestrogens, may influence the subsequent development of breast cancer in offspring 2 by altering the hormonal environment of the developing foetal mammary glands. ${ }^{3}$ Oestrogen acts as a key driver for breast cancer because it can increase the frequency of mitotic activity, thereby stimulating cell division and increasing the potential of cells to accumulate mutations. ${ }^{4}$ Mammary gland cells in utero are not terminally differentiated. Therefore, factors that increase the risk of cancer during adult life (such as exogenous and endogenous oestrogens in breast cancer) may have similar effects when they act in utero. ${ }^{5}$

The ratio between the length of the second and fourth digit, known as digit ratio or 2D:4D, has been considered a putative biomarker for prenatal hormonal exposure mainly exposure to testosterone and oestrogen. ${ }^{6}$ Digit ratio is negatively correlated to prenatal testosterone and positively correlated to prenatal oestrogen, ${ }^{6-8}$ and has been used as a biomarker for several diseases. ${ }^{9}$ In oncology, it was suggested that 2D:4D may be predictive of susceptibility to different types of cancers, ${ }^{10}$ like

Corresponding author: Patrícia Helena Costa Mendes; Department of Health Science Program, State University of Montes Claros, Minas Gerais State, Brazil.

Cite this article as: Mendes PH, Gomes AC, Soares PB, Gonçalves E, Macedo C, Silveira M, Martelli D, Paranaíba LM, Martelli-Júnior H. Digit ratio (2D:4D) is associated with breast cancer. Int J Cancer Ther Oncol. 2016; 4(3):438. DOI: 10.14319/ijcto.43.8 
prostate cancer, ${ }^{\mathbf{1 1 - 1 3}}$ breast cancer, ${ }^{\mathbf{1 4 - 1 6}}$ gastric cancer, ${ }^{17}$ colorectal cancer, ${ }^{18}$ and oral cancer. ${ }^{19}$

There is evidence that genes involved in the differentiation of foetal 2D:4D may also be involved in mammary gland initiation and breast carcinogenesis events. During the development of 2D:4D, there are at least 19 skeletogenic genes that are activated or deactivated by prenatal testosterone and prenatal oestrogen. ${ }^{20}$ Among them, there are genes (WNTS and FGFR) that influence the TBX genes, which initiate mammary gland formation. ${ }^{21}$ Homeobox (HOX) family genes, which are essential for the differentiation of fingers, ${ }^{7}$ are related to breast carcinogenesis. HOXA5 limits the p53 expression in breast tumors ${ }^{22}$ and HOXA1 represents a human mammary epithelial oncogene. ${ }^{23}$ Furthermore, the SOX9 gene, which also acts during development of 2D:4D, ${ }^{20}$ might be important for breast tumour initiation and metastasis. ${ }^{24}$

Breast cancer is the most common malignancy, comprising $23 \%$ of all cancer cases worldwide. ${ }^{25}$ In Brazil, it was estimated that there would be 57,120 new cases of breast cancer by 2014 and in 2011, had 13,225 women died from it. Breast cancer, if diagnosed and treated in a timely manner, it has a good prognosis, which reinforces the importance of implementing preventive measures for cancer control and improved survival. ${ }^{26}$ Thus, the aim of this study is to investigate whether 2D:4D is a marker for breast cancer in a Brazilian population by comparing 2D:4D ratios among women with and without breast cancer and to inquire about the relationship between 2D:4D and the age at diagnosis of the disease.

\section{Methods and Materials}

\subsection{Samples}

A case-control study was performed between September 2013 and October 2014 with a total of 100 patients with breast cancer (BCA group) and 100 healthy controls. Patients with breast cancer were recruited from the Oncology Department of Santa Casa, Montes Claros, Minas Gerais State, Brazil, who had a confirmed diagnosis of invasive adenocarcinoma of the breast by histopathological report. Women with in situ breast cancer were not included.15 The controls were chosen from healthy women, age-matched to the other group. After identifying the cases, control women were selected from the medical records of patients from health public services seen annually by nurses and gynaecologists for the prevention of breast and uterus cancers. Exclusion criteria for both groups involved a history of fractures on fingers of either hand and hormonal disorders. Written informed consents were obtained, and the study was carried out with approval of the Human Research Ethics Committee of the University (311.756/2013).

\subsection{D:4D ratio}

The length of the second (index) and fourth (ring) fingers were measured by the direct method using digital Vernier callipers with a resolution of $0.01 \mathrm{~mm}$. Direct method was used instead of the indirect technique (from photocopies or scans), as the latter tends to show some systematic distortion. ${ }^{27}$ Measurements were taken from the tip of the finger to the basal crease and were repeated two times, with a 30-minute interval between measurements. The second measurement was blind to the first. The analysed ratio was the mean of the two performed measurements. The measurements were undertaken by a unique researcher. When two creases were visible at the base of the digit, the crease proximal to the palm was chosen. The length of the index finger was divided by the length of the ring finger to obtain the 2D:4D ratio. These were identified right hand digit ratio (R2D4D), left hand digit ratio (L2D4D) and the difference between the right and left 2D:4D known as DR - L. The right minus left 2D:4D was calculated as the difference between the right hand and left hand 2D:4D (DR-L). ${ }^{15}$ Intraclass correlation coefficients (ICCs) were calculated to check intraobserver reliability of the R2D, R4D, R2D4D, L2D, L4D, L2D4D, and DR-L measurements. ${ }^{6}$

\subsection{Statistical analysis}

Sample size was calculated according to a specific formula for sample calculation using averages of independent groups. ${ }^{28}$ A significance level of $5 \%$ and power of $80 \%$ was adopted. The mean values and standard deviations used in the sample formula was based on the results of a pilot study previously conducted with 15 subjects in each group, with an observed mean (and standard deviation) of 2D:4D in the BCA group of $0.98( \pm 0.03)$ and $0.96( \pm 0.03)$ for the healthy subjects. Thus, for this study we would require at least 36 individuals in each group.

For the analysis of results, a descriptive statistical analysis was initially carried out. The general characteristics of groups such age, age at menarche, alcohol consumption, smoking habits, and hormone replacement therapy were compared between groups by the Chi-square tests. The Shapiro Wilk test was used to check the distribution of the samples and revealed that right 2D:4D and left 2D:4D follow a normal distribution, but DR-L and age at diagnosis of breast cancer did not follow a normal distribution. Then, subsequently a Student's $t$-test for unpaired samples and Mann-Whitney test were performed to compare the means of 2D:4D ratios and DR-L among groups. To examine whether there is any relationship between 2D:4D measurements and age at diagnosis of breast cancer, we employed Spearman`s Correlation. All analyses were conducted using SPSS 19.0 for Windows (IBM SPSS, Armonk, NY USA) with a significance level of $5 \%$. 


\section{Results}

Intraobserver reliability was high for raw digit measurements, with ICC for left and right index and ring fingers all being greater than 0.98 . ICC for 2D:4D ratio and DR-L were lower than those for raw digit measurements $(0.94$ for right 2D:4D, 0.96 for left 2D:4D and 0.87 for DR-L), but still suggest that the observed variability in digit ratio is due to individual differences rather than measurement error.

Table 1: Characteristics of study population.

\begin{tabular}{lccc}
\hline \hline Characteristics & $\begin{array}{c}\text { BCA Group } \\
\text { Mean }(s d)\end{array}$ & $\begin{array}{c}\text { Control Group } \\
\text { Mean }(s d)\end{array}$ & $p$-value \\
\hline Age & $55.63(11.60)$ & $55.48(11.57)$ & 1 \\
Age at menarche & $13.57(1.41)$ & $13.19(1.93)$ & 1 \\
& $\mathrm{n}$ & $\mathrm{N}$ & \\
$T C^{*}$ & 79 & 80 & $0.325^{a}$ \\
Never & 20 & 16 & \\
Ex-smokers & 01 & 04 & \\
Smokers & & & \\
EC & 79 & 85 & \\
Never & 11 & 10 & \\
Ex-drinkers & 10 & 05 & \\
Drinkers & & & $0.350^{a}$ \\
HT & 07 & 96 & \\
Yes & 93 & & \\
No & & & \\
\hline \hline
\end{tabular}

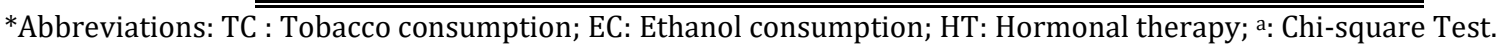

Table 2: Comparison of right hand digit ratio (R2D4D), left hand digit ratio (L2D4D) and right hand minus left hand digit ratio (DR-L) between patients with breast cancer (BCA Group) and Control Group.

\begin{tabular}{ccccccc}
\hline \hline & $\begin{array}{c}\text { BCA Group } \\
\text { Mean }(s d)\end{array}$ & CI95\% & $\begin{array}{c}\text { Control Group } \\
\text { Mean }(s d)\end{array}$ & CI95\% & $\begin{array}{c}p \text { - } \\
\text { value }\end{array}$ & Size effect \\
\hline R2D4D & $0.9803(0.0353)$ & $(0.9733-0.9873)$ & $0.9499(0.0336)$ & $(0.9432-0.9566)$ & $0.001^{a}$ & $r=0.16$ \\
L2D4D & $0.9770(0.0336)$ & $(0.9704-0.9837)$ & $0.9562(0.0370)$ & $(0.9488-0.9635)$ & $0.001^{a}$ & $r=0.28$ \\
DR-L & $0.0033(0.0720)$ & $(-0.0022-0.0087)$ & $-0.0063(0.0345)$ & $(-0.0131-0.0005)$ & $0.032^{b}$ & $r=0.14$ \\
\hline \hline
\end{tabular}

a: Student's t-test for unpaired samples; b: Mann-Whitney test.

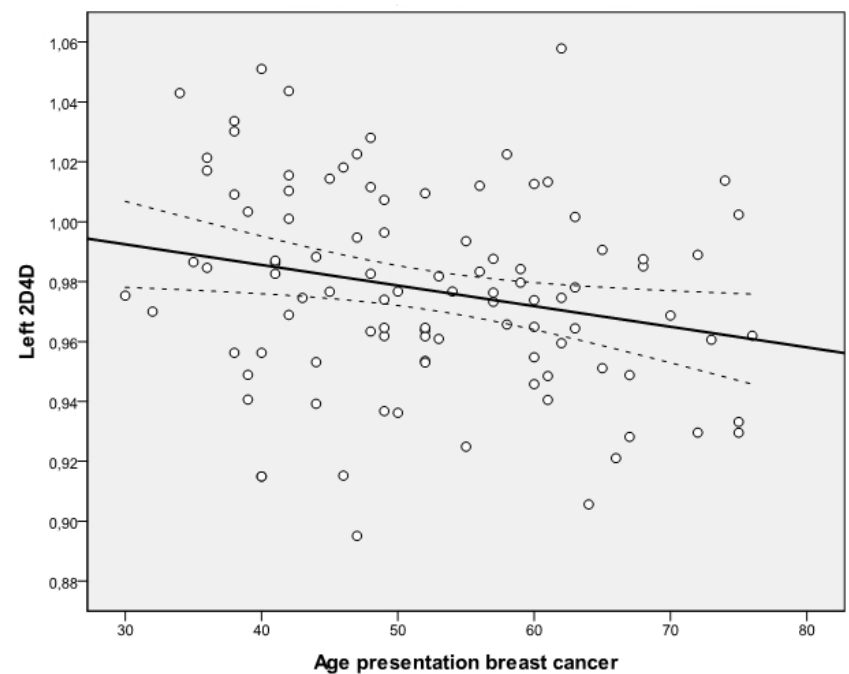

Figure 1: Scatter plot showing the negative correlation between age at presentation of breast cancer and left hand 2D:4D in the group with breast cancer. 
The age of the women in both groups ranged from 30 to 82 years old and the age at diagnosis of breast cancer (in BCA group) ranged from 30 to 76 years old with a mean age of 52 years $( \pm 11.46)$. Characteristics of the study population such as age, age at menarche, tobacco, alcohol consumption, and hormonal therapy are shown in Table 1. In our sample, women with breast cancer presented significantly higher R2D4D, L2D4D, and DR-L than controls, as shown in Table 2.

Regarding the association between digit ratio measurements and age at diagnosis of breast cancer in the BCA group, an inverse association between L2D4D and age at diagnosis of breast cancer was noted ( $\mathrm{r}=$ $-0.246, p=0.014$; Figure 1). On the other hand, no correlation was found between R2D:4D and DR-L with age at diagnosis of breast cancer.

\section{Discussion}

There is some evidence that high concentrations of endogenous oestrogen in utero may increase the risk for subsequent breast cancer ${ }^{2}$ and that high sensitivity to prenatal oestrogen exposure was correlated with high 2D:4D. ${ }^{6}$ Therefore, high 2D:4D has a positive correlation with risk of breast cancer. The hypothesis that 2D:4D should be positively associated with breast cancer was first put forward by Manning and Leinster ${ }^{\mathbf{1 4}}$ and was also expanded upon by Manning. ${ }^{29,30}$ Our findings provide support to this hypothesis, since they showed significant differences in 2D:4D on both hands between groups, with breast cancer patients having a higher 2D:4D than controls. Our results are in agreement with another study conducted later by Hong et al. ${ }^{16}$ who also observed that women with breast cancer presented a higher 2D:4D of both hands than a group of healthy women.

The fact that both hands have shown significant differences point to the importance of including the left hand in studies evaluating 2D:4D and cancer. This statement is emphasised by Hopp et al. ${ }^{17}$ who also noted significant differences in the left hand of individuals with gastric $^{17}$ and colorectal cancer ${ }^{18}$ when compared with control subjects. Muller et al. ${ }^{15}$ also observed an association between left hand values and the risk of breast cancer. Several studies conducted earlier on this perspective evaluated only the right hand ${ }^{12,13,19,31,32}$ based on the assumption that the right hand has more sensitivity to prenatal hormones. ${ }^{33}$

Other relevant data of this study includes that DR-L presented a significant difference between the groups: women with breast cancer had a greater difference in relation to controls. There is evidence indicating that DR-L is inversely associated with prenatal exposure and sensitivity to testosterone. ${ }^{33,34}$ Thus, the observed result is in agreement, since the difference was higher in the group with cancer, indicating a lower exposure to testosterone and increased exposure to oestrogen. This has the ability to directly stimulate the proliferation of breast cells and could affect the number of mammary gland cells at transformation, which increases the risk of breast cancer. ${ }^{5}$ Inversely, Muller et al. ${ }^{\mathbf{1 5}}$ observed a relationship between low DR-L and a higher risk of breast cancer and explained this finding by suggesting that androgens may exert any proliferative effects indirectly after conversion to oestrogen by the aromatase enzyme.

The present study also showed that women with breast cancer who have higher 2D:4D presented breast cancer earlier than women with lower 2D:4D ratios (Figure 1). This finding was especially found in the left hand, which showed an inverse and significant association with age at diagnosis of breast cancer. Our results are consistent with Manning and Leinster ${ }^{14}$ and Hong et al. ${ }^{16}$, who also demonstrated an inverse association between left 2D:4D and age when cancer presented. On the other hand, Muller et al. ${ }^{15}$ reported that both right 2D:4D and DR-L were inversely related to age of diagnosis. Neither Manning and Leinster ${ }^{14}$ nor Hong et al. ${ }^{16}$ explained why the effect was stronger in the left hand, whereas scientific evidence would expect a stronger association in relation to the right hand. Hong et al. ${ }^{16}$ suggest that the reason for these different results may be ethnic differences or due to sample size. In fact, in relation to the sample size, the number of women in this study was similar to those included in the studies by Manning and Leinster ${ }^{\mathbf{1 4}}$ and Hong et al. ${ }^{\mathbf{1 6}}$ Regardless, these conflicting findings reinforce the importance of evaluating both hands in studies assessing digit ratio and cancer.

There are large ethnic differences in 2D:4D. Therefore, the ethnic variation in $2 \mathrm{D}: 4 \mathrm{D}$ is of methodological importance, as it implies that ethnicity needs to be assessed and controlled in 2D:4D studies that should carefully match participants for ethnicity or study ethnically homogeneous samples. ${ }^{29}$ Within this context, it is important to make some remarks about the Brazilian population's race. The Brazilian population was formed by extensive admixture from three different ancestral roots: Amerindians, Europeans, and Africans. This resulted in a great variability of skin pigmentation, with no discontinuities between Black and White skin color. ${ }^{35}$ Thus, the race of Brazilian individuals cannot be determined by skin colour. The ideal method is to genetically identify the contribution of components of ancestry to characterise the race of the study population. Several studies conducted in 38 different Brazilian populations, including the population of the state of the present study, concluded that European ancestry is the major contributor to the genetic background of Brazilians ${ }^{36}$, which approximates the racial characteristics of this study population with those of the aforementioned studies. Nevertheless, the heterogeneity of the Brazilian race can be considered an important limitation in Brazilian studies of 2D:4D. 
Some known risk factors for breast cancer were measured in this study, such as hormone replacement therapy, alcohol consumption, smoking habits, and age at menarche. These factors did not differ between the two groups. Such risk factors reinforce the importance of the cumulative effect of exposure to oestrogen in the aetiology of breast cancer. ${ }^{37}$ Since in this study the distributions of these conditions did not differ between groups, it underscores the role of prenatal oestrogen exposure on the subsequent risk of developing breast cancer.

\section{Conclusion}

Our results provide additional evidence that strengthens the relationship between $2 \mathrm{D}: 4 \mathrm{D}$ as a putative biomarker associated with breast cancer, showing that high 2D:4D is associated with higher intrauterine exposure to oestrogens, which in turn acts to increase the risk of breast cancer. Moreover, higher 2D:4D is associated with earlier onset of the disease.

As this association is still incipient due to the low number of published studies, further research is needed in different populations in order to confirm this correlation. The relevance of validating 2D:4D as a marker for breast cancer could also help, in addition to the research of risk factors and as an auxiliary strategy in primary prevention measures (warning to possible modifications in the breast, changes in lifestyle that reduces exposure to risk factors) and secondary measures such as early involvement in screening tests for women who have a digital pattern of greater susceptibility.

\section{Conflict of interest}

The authors declare that they have no conflicts of interest. The authors alone are responsible for the content and writing of the paper.

\section{Funding}

This work was supported by grants from Minas Gerais State Research Foundation - Fapemig, Brazil; National Council for Scientific and Technological Development CNPq, Brazil; Coordination of Improvement of Higher Education Personnel (CAPES), Brazil and Procad/Casadinho - CAPES/CNPq.

\section{References}

1. Potischman N, Troisi R, Vatten L. The life course approach to cancer epidemiology. In: Kuh D, Ben-Shlomo Y, editors. A life course approach to chronic disease epidemiology. New York: Oxford University Press;2004.
2. Trichopoulos D. Hypothesis: does breast cancer originate in utero. Lancet. 1990;335(8695): 939-40.

3. Park SK, Kang D, McGlynn KA, et al. Intrauterine environments and breast cancer risk: meta-analysis and systematic review. Breast Cancer Res. 2008;10(1):1-8.

4. Yager JD, Davidson NE. Estrogen carcinogenesis in breast cancer. N Engl J Med. 2006; 354(3): 270-82.

5. Trichopoulos D. Intrauterine environment, mammary gland mass and breast cancer risk. Breast Cancer Res. 2003;5(1):42-4.

6. Manning JT, Scutt D, Wilson J, Lewis-Jones DI. The ratio of $2^{\text {nd }}$ to $4^{\text {th }}$ digit length: a predictor of sperm numbers and concentrations of testosterone, luteinising hormone and oestrogen. Hum Reprod. 1998;13(11):3000-4.

7. Manning JT, Callow M, Bundred PE. Finger and toe ratios in humans and mice: implications for the aetiology of diseases influenced by HOX genes. Med Hypotheses. 2003;60(3):340-3.

8. Breedlove SM. Minireview: Organizational hypothesis: instances of the fingerpost. Endocrinology 2010;151(9):4116-22.

9. Manning JT, Bundred PE. The ratio of $2^{\text {nd }}$ to $4^{\text {th }}$ digit length: a new predictor of disease predisposition? Med Hypotheses. 2000;54(5):855-7.

10. Hopp RN, Lima N, Filho J, et al. Digit Ratio (2D:4D) and cancer: What is known so far? Int J Cancer Ther Oncol. 2014; 2(1):020111.

11. Mendes PHC, Martelli DRB, Costa SM, et al. Comparison of digit ratio (2D:4D) between Brazilian men with and without prostate cancer. Prostate Cancer Prostatic Dis. 2016;19(1):107-10.

12. Jung H, Kim KH, Yoon SJ, Kim TB. Second to fourth digit ratio: a predictor of prostate-specific antigen level and the presence of prostate cancer. BJU Int. 2010;107(4):591-6.

13. Rahman AA, Lophatananon A, Stewart-Brown S, Harriss D, Anderson J, Parker T, et al. Hand pattern indicates prostate cancer risk. $\mathrm{Br} \mathrm{J}$ Cancer. 2011;104(1):175-7.

14. Manning JT, Leinster S. The ratio of $2^{\text {nd }}$ to $4^{\text {th }}$ digit length and age at presentation of breast cancer: a link with prenatal oestrogen. Breast. 2001;10(4):355-7.

15. Muller DC, Baglietto L, Manning JT, et al. Second to fourth digit ratio (2D:4D), breast cancer risk factors, and breast cancer risk: a prospective cohort study. Br J Cancer. 2012; 107(9):1631-6.

16. Hong L, Zhan-Bing M, Zhi-Yun S, et al. Digit ratio (2D:4D) in Chinese women with breast cancer. Am J Hum Biol. 2014;26(4):562-4.

17. Hopp RN, Lima NCDS, Filho JLF, et al. Digit ratio (2D:4D) is associated with gastric cancer. Early Hum Develop. 2013;89(5):327-9. 
18. Hopp RN, Lima NCDS, Filho MS, et al. Digit ratio is associated with colorectal cancer. J Gastrointest Dig Syst. 2015;5(1):1-14.

19. Hopp RN, Jorge J. Right hand digit ratio (2D:4D) is associated with oral cancer. Am J Hum Biol. 2011; 23(3):423-5.

20. Zheng Z, Cohn MJ. Developmental basis of sexually dimorphic digit ratios. Proc Natl Acad Sci USA. 2011;108(39):16289-94.

21. Eblaghie MC, Song SJ, Kim JY, et al. Interactions between FGF and Wnt signals and Tbx3 gene expression in mammary gland initiation in mouse embryos. J Anat. 2004;205(1):1-13.

22. Raman V, Martensen SA, Reisman D, et al. Compromised HOXA5 function can limit p53 expression in human breast tumours. Nature. 2000;405(6789):974-8.

23. Zhang X, Zhu T, Chen Y, et al. Human Growth hormone-regulated HOXA1 is a human mammary epithelial oncogene. J Bio Chem. 2003;278(9):7580-90.

24. Chakravarty G, Moroz K, Makridakis NM, et al. Prognostic significance of cytoplasmic SOX9 in invasive ductal carcinoma and metastatic breast cancer. Exp Biol Med. 2011; 236(2):145-55.

25. Jemal A, Bray F, Center MM, et al. Global cancer statistics. CA Cancer J Clin. 2011;61(2):69-90.

26. National Cancer Institute. Estimate 2014: incidence of cancer in Brazil. National Cancer Institute José Alencar Gomes da Silva: Rio de Janeiro, Brazil, 2014.

27. Ribeiro E, Neave N, Morais RN, Manning JT. Direct versus indirect measurement of digit ratio (2D:4D); a clinical review of the literature and new data. Evol Psychol. 2016;14(1):1-8.
28. Arango HG. Biostatistics - Theoretical and computational. Nova Guanabara: Rio de Janeiro, Brazil, 2009.

29. Manning JT. Digit ratio: a pointer to fertility, behaviour and health. New Brunswick: Rutgers University Press, 2002.

30. Manning JT. The finger ratio. London: Faber and Faber, 2008.

31. Waters M, Rebholz CM, Wood B, et al. Second to fourth digit ratio and prostate cancer severity. Prostate Cancer Prostatic Dis. 2013;16(1):107-10.

32. Hopp RN, Jorge J. Right hand digit ratio (2D:4D) is associated with prostate cancer: Findings of an admixed population study. J Solid Tumors. 2012;2(1):22-5.

33. Hönekopp J, Watson S. Meta-analysis of digit ratio 2D:4D shows greater sex difference in the right hand. Am J Hum Biol. 2010;22(5):619-30.

34. Hurd PL, Vaillancourt KL, Dinsdale NL. Aggression, digit ratio and variation in androgen receptor and monoamine oxidase a genes in men. Behav Genet. 2011;41(4):543-6.

35. Pena SD, Di Pietro G, Fuchshuber-Moraes M, et al. The genomic ancestry of individuals from different geographical regions of Brazil is more uniform than expected. PLoS One. 2011; 6(2):e17063.

36. Moura RR, Coelho AVC, Balbino VQ, et al. Meta-analysis of Brazilian genetic admixture and comparison with other Latin America countries. Am J Hum Biol. 2015;27(5):674-80.

37. Henderson BE, Feigelson HS. Hormonal carcinogenesis. Carcinogenesis 2006;21(3):427-33. 